\title{
Designing Interaction for Next Generation Personal Computing
}

\author{
Giorgio De Michelis ${ }^{1,2}$, Marco Loregian ${ }^{1,2}$, Claudio Moderini ${ }^{1,3}$, Patrizia Marti ${ }^{4}$, \\ Cesare Colombo ${ }^{5}$, Liam Bannon ${ }^{6}$, Cristiano Storni ${ }^{6}$, and Marco Susani ${ }^{7}$ \\ ${ }^{1}$ ITSME srl, viale Sarca 336-F, 20126 Milano, Italy \\ \{giorgio.demichelis, marco. loregian, claudio.moderini\}@itsme.it \\ ${ }^{2}$ University of Milano-Bicocca, viale Sarca 336-F, 20126 Milano, Italy \\ \{gdemich, loregian\} @disco.unimib.it \\ ${ }^{3}$ Domus Academy, Via Watt 27, 20143 Milano, Italy \\ claudio.moderini@domusacademy. it \\ ${ }^{4}$ Communication Science Dept., University of Siena, Via Roma 56, 53100 Siena, Italy \\ marti@unisi.it \\ ${ }^{5}$ CEFRIEL, Politecnico of Milano, Via Fucini 2, 20133 Milano, Italy (Italy) \\ colombo@cefriel.it \\ ${ }^{6}$ University of Limerick, Ireland \\ \{liam.bannon, cristiano.storni\}@ul.ie \\ ${ }^{7}$ Motorola Mobile Devices, Chicago, USA \\ marco.susani@motorola.com
}

\begin{abstract}
Over two decades of research in the field of Interaction Design and Computer Supported Cooperative Work convinced us that the current design of workstations no longer fits users' needs. It is time to design new personal computers based on metaphors alternative to the desktop one. With this SIG, we are seeking to involve international HCI professionals into the challenges of designing products that are radically new and tackling the many different issues of modern knowledge workers. We would like to engage a wider cross-section of the community: our focus will be on issues of development and participation and the impact of different values in our work.
\end{abstract}

\section{Introduction}

With the emergence of new interaction paradigms, such as cloud, mobile and ubiquitous computing, and the evolution of personal computing on the Web, e.g., due to the success of Social Computing (Web 2.0) and to the evolution of Semantic Web technologies, it is now clear that the desktop metaphor is obsolete. Actually, research literature sustaining this claim is vast and nearly as old as the metaphor itself - which was developed by Alan Kay and colleagues at the Xerox PARC in the late "70s and reached the market in 1984 with the Apple Macintosh.

Researchers in the various fields of computer science (computer supported cooperative work, ubiquitous computing, knowledge management, cooperative information systems, etc.) and design (interaction design, human computer interaction, etc.) have tackled the problem of finding better ways to support users in interacting with 
computers from many perspectives, bringing forth new ways to look at work practices, better understanding of the relations between language and action, and a closer attention to knowledge sharing within collaborative teams and communities of practice. The focus has been for long time on the shared services that could be provided to people: only in the last years, researchers and practitioners started to pay attention to the way personal workstations could be redesigned in order to go beyond the desktop metaphor and become better tools to help users to manage the complexity of their manifold activities.

This SIG aims at fostering a cross-cultural discussion on how to design interaction for next-generation personal computing, especially taking into consideration the aspects of situatedness of actions.

\section{Attendance and Format of Discussions}

This SIG is for two groups of attendees - those with some experience of working in this field, e.g., who already tried to develop innovative interactive systems, who want to compare and share practice, and others with little or no experience, who are interested in learning more and possibly starting new projects and finding collaborations.

The SIG will start from a brief introduction of the itsme project $^{1}$, in which most of the organizers are involved, and on the motivations and features of the design approach. The metaphor of stories and venues will be introduced and analyzed as a case of alternative to the desktop metaphor. As already happened in previous seminars and tutorials, this introduction will start a discussion on issues such as:

- The role of the Web in the design of personal computing for the future, with particular attention to social and semantic aspects;

- The role between research and industry in developing anr releasing innovative products on the market.

The duration of a SIG will not allow for a very detailed sharing or debate on personal experiences; therefore, we see this as an important beginning to ongoing process. We plan to continue the conversation online, actively involving the participants in the itsme community, in which the issues of open innovation are of foremost importance.

\footnotetext{
${ }^{1}$ http://www.itsme.it
} 